\title{
Société suisse de chirurgie thoracique et cardio-vasculaire (SSCTCV)
}

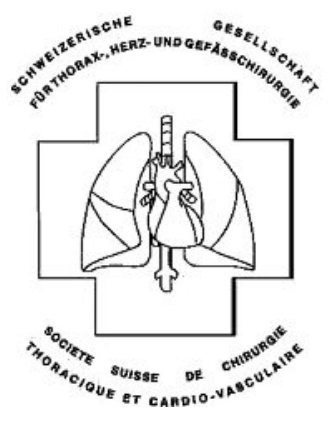

Peter Stierli ${ }^{a}$, Thierry Carrel $^{b}$

a Prof. Dr, président SSCTCV, Médecin-chef du service de chirurgie vasculaire, Hôpital cantonal d'Aarau

b Prof. Dr, secrétaire a.i., Directeur de la Clinique universitaire de chirurgie cardiovasculaire,

Hôpital de l'Ile de Berne
Fondée en 1986, la Société suisse de chirurgie thoracique et cardio-vasculaire (ci-après SSCTCV) est une association professionnelle et une société scientifique. La SSCTCV regroupe des spécialistes et des scientifiques qui s'occupent des maladies du thorax, du cœur et des vaisseaux.

Depuis la fondation de la société, la spécialisation croissante des domaines chirurgicaux a continué à prendre de l'ampleur. Le domaine «chirurgie cardiaque et vasculaire thoracique» a introduit en 1991 un titre de spécialiste tandis que les disciplines de la chirurgie vasculaire et de la chirurgie thoracique sont devenues en 2001 des formations approfondies pouvant être acquises aussi bien par les détenteurs du titre de spécialiste en «chirurgie générale» que par ceux en «chirurgie cardiaque et vasculaire thoracique». Les deux disciplines, la chirurgie vasculaire et la chirurgie thoracique, ont en outre fondé leur propre société de formation approfondie (SSCV et SSCT). de même que pour les formations approfondies en chirurgie thoracique et chirurgie vasculaire.

- Promouvoir la collaboration avec des représentants nationaux et internationaux des disciplines analogues et apparentées.

- Garantir la qualité de l'activité médicale; défendre les intérêts professionnels et les principes de base éthiques de l'activité médicale exercée par ses membres. A cet effet, la société s'appuie essentiellement sur le code de déontologie de la FMH.

La SSCTCV est représentée à la Fondation Suisse de Cardiologie, à l'association Cardiovasc Suisse, à la fmCh et à la Chambre médicale.

Sur le plan de la politique professionnelle, la Société suisse de chirurgie vasculaire (SSCV) et la Société suisse de chirurgie thoracique (SSCT) sont représentées aussi bien par la Société suisse de chirurgie (SSC) que par la Société suisse de chirurgie thoracique et car-

\section{Les manifestations annuelles communes donnent l'occasion aux chirurgiens des différentes disciplines spécialisées de se rencontrer et d'éviter toute nouvelle scission de la chirurgie}

Dans les cliniques universitaires de Berne, Genève, Lausanne et Zurich, la chirurgie cardiaque et la chirurgie vasculaire sont exercées dans une clinique commune; au CHUV à Lausanne, la chirurgie vasculaire est en outre offerte aussi par le service de chirurgie thoracique et vasculaire de la clinique de chirurgie générale. A l'Hôpital universitaire de Bâle, la chirurgie cardiaque est exercée sous forme de discipline isolée, tandis que la chirurgie vasculaire est exercée dans le cadre d'une coopération avec l'Hôpital cantonal d'Aarau.

La société compte aujourd'hui 170 membres qui proviennent principalement du domaine de la chirurgie cardiaque.

La SSCTCV assume les tâches suivantes:

Correspondance: Prof. Dr Peter Stierli Kantonsspital Aarau Buchserstrasse

CH-5001 Aarau Tél. 0628384513

peter.stierli@ksa.ch
- Promouvoir les sciences et la recherche dans le domaine des maladies cardiovasculaires et thoraciques; soutenir l'enseignement dans le cadre de la formation prégraduée, postgraduée et continue.

- Préparer une relève qualifiée pour le titre de spécialiste en chirurgie cardiaque et vasculaire thoracique dio-vasculaire. Cette double représentation correspond au monde hospitalier réel dans lequel la chirurgie vasculaire est exercée aussi bien par des spécialises en chirurgie cardiaque que par des spécialistes en chirurgie générale.

Pour éviter dans notre petit pays une pléthore de congrès organisés par des sociétés de taille relativement petite, la SSC et la SSCTCV organisent ensemble leur congrès annuel depuis sept ans. Outre l'efficacité de l'organisation, les manifestations annuelles communes donnent l'occasion aux chirurgiens des différentes disciplines spécialisées de se rencontrer et d'éviter toute nouvelle scission de la chirurgie.

La SSCTCV dispose depuis peu d'un site Internet (www.sgthgc.ch), prévu aussi bien pour ses membres que pour un large public. Depuis 1996, elle publie son propre organe officiel, intitulé «Médecine cardiovasculaire». 